\title{
A!
}

This is an electronic reprint of the original article.

This reprint may differ from the original in pagination and typographic detail.

Haarla, Jaakko; Lehtovuori, Anu; Viikari, Ville

\section{Base station antenna array with calibration structure}

\section{Published in:}

12th European Conference on Antennas and Propagation (EuCAP 2018)

DOI:

10.1049/cp.2018.0368

Published: 01/01/2018

Document Version

Peer reviewed version

Please cite the original version:

Haarla, J., Lehtovuori, A., \& Viikari, V. (2018). Base station antenna array with calibration structure. In 12th

European Conference on Antennas and Propagation (EuCAP 2018) https://doi.org/10.1049/cp.2018.0368

This material is protected by copyright and other intellectual property rights, and duplication or sale of all or part of any of the repository collections is not permitted, except that material may be duplicated by you for your research use or educational purposes in electronic or print form. You must obtain permission for any other use. Electronic or print copies may not be offered, whether for sale or otherwise to anyone who is not an authorised user. 


\title{
Base Station Antenna Array with Calibration Structure
}

\author{
Jaakko Haarla, Anu Lehtovuori, Ville Viikari \\ Department of Electronics and Nanoengineering, Aalto University, Espoo, Finland, jaakko.haarla@aalto.fi
}

\begin{abstract}
The introduction of 5G technologies is pushing research in mobile communications to millimeter waves. However, sub-6 GHz frequency range as a part of the $5 \mathrm{G}$ standard provides excellent wave propagation properties and more capacity due to new usable frequency ranges. This paper introduces a design for picocell base station $2 \times 2$ antenna array with integrated signal injection network for transceiver calibration. Operation frequency range is from 2 to $6 \mathrm{GHz}$. The antenna array with calibration board has better than $-6.3 \mathrm{~dB}$ active reflection coefficient and efficiency higher than $\mathbf{- 3} \mathrm{dB}$. Calibration network provides $29 \mathrm{~dB}$ coupling.
\end{abstract}

Index Terms-Base station, beamforming, calibration network, mobile communications, sub-6 GHz.

\section{INTRODUCTION}

According to the Cisco VNI forecast, the compound annual growth rate of mobile data between years 2016 and 2022 is $46 \%$ [1]. This brings challenges to the mobile communication technologies: How to fulfill the ever growing need for higher data throughput? Implementation of 5G standard will offer the solution [2]. With 5G, there has been a discussion on sharing allocated frequency bands which are already used by other applications [3]. In 5G, these Long-Term Evolution (LTE) bands could be combined with WiFi frequency allocations to increase data rates and efficiency of the radio spectrum usage [4].

In addition, sub- $6 \mathrm{GHz}$ frequencies propagate better inside buildings than millimeter waves and do not require line-ofsight [5]. One foreseen trend in $5 \mathrm{G}$ is network densification: Small picocells are placed to offices and crowd areas outdoors. Therefore it is important that these base stations are imperceptible and small not affecting normal usage of e.g. office space.

$5 \mathrm{G}$ technologies will utilize electronically steerable antenna arrays. One challenge of phased antenna arrays is potential drifting of phase shifters. Drifting is not typically an issue in reception, because possible drift can easily be calibrated out by adjusting phase shifters to maximize the received power. However, such an easy calibration method can not be utilized in transmission. If the drift is too large, transmitted signal may be directed off from the receiver. To avoid that, a selfcalibration capability can be realized by feeding known signal to all transceiver ports and measuring the difference between the signals [6].

In this paper, we showed the implementation of a picocell antenna array in order to study beam steering in the sub- $6 \mathrm{GHz}$ frequency range. The goal is to design an antenna array with a calibration network which could also include at least part of

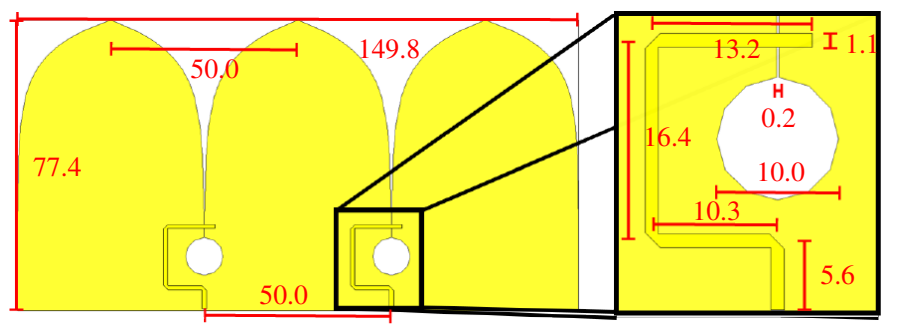

Figure 1. Vivaldi antenna PCB and balun structure. All dimensions are in $\mathrm{mm}$.

the electronics for the picocell base station and function also as a base structure. The frequency range is from 2 to $6 \mathrm{GHz}$.

This paper is divided as follows: Section II discusses the details of the design. Introduction of prototype, results from the EM simulations and prototype measurements are presented in Section III. Conclusions from this design are discussed in Section IV.

\section{PROPOSED ANTENNA ARRAY DESIGN}

The antenna array is designed for a 4-channel transceiver IC. The proposed antenna array design consists of signal distribution printed circuit board (PCB) and two antenna array PCBs. Signal distribution board includes signal paths from the IC to antenna feeds and also the calibration network from calibration signal output to the IC RF-port with equal amplitude and phase. The overall design includes four antennas, four signal traces, and calibration network. These are discussed in more detail in II-A, II-B, and II-C.

\section{A. Antenna array}

Vivaldi antenna was chosen for the design due to its ultra wide frequency band and directivity [7]. Both antenna PCBs include two Vivaldi antennas in the middle and an additional parts on the sides as shown in Fig. 1. The additional parts on the sides are used to improve matching below $3 \mathrm{GHz}$, where the half-wavelength is larger than the opening of the Vivaldi antenna.

Antenna element width (and also element spacing) is a trade-off between the impedance bandwidth and grating-lobe free beam steering range. As a compromise, we chose $50 \mathrm{~mm}$ element separation distance. Wider antenna spacing would enable lower cutoff frequency for the antenna and decrease the mutual coupling. However, at higher frequencies the grating 


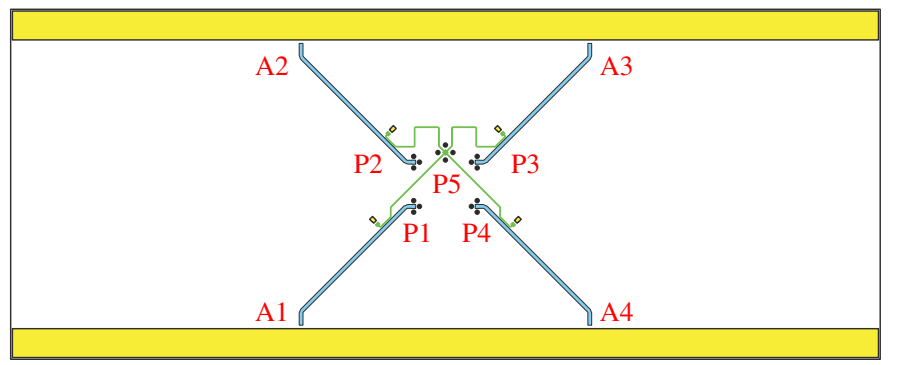

Figure 2. Distribution board with port (P) and antenna (A) locations. Calibration network is highlighted in green and Main RF lines in blue.

lobes would move closer to the main beam and steering capability would be degraded.

The curve of Vivaldi antenna was calculated using equations presented in [8] with the following parameters: $\left(x_{1}, y_{1}\right)=$ $(0,0.1),\left(x_{2}, y_{2}\right)=(50,25)$ and $r=0.11$. The separation between Vivaldi elements is $0.2 \mathrm{~mm}$. Antenna is fed with a sickle shaped $50 \Omega$ microstrip line which works as a balun [9].

\section{B. IC location and feeding network}

One PCB is used as a base for the array. Upper side of the PCB is dedicated to RF-traces while the other side is reserved for power distribution and logic. RF IC transceiver would locate in the middle of the back side of the PCB to utilize the symmetry of the design. RF signals are routed through vias from one side to the other. In the middle of the PCB, there is a ground plane to isolate these two sides from each other.

The main RF line is identical in all four channels. The line is a $50 \Omega$ microstrip that is fed with vias through the PCB to the IC-side and other end is open so that antenna PCBs can be attached to it.

\section{Calibration network}

The calibration board connections seen in Fig. 2 can be split into three functional sections: Directional coupler, Main RF line, and Calibration network.

Fig. 3a shows the directional coupler used between the calibration network and each Main RF Line. This couples the signal fed from the calibration port to the Main RF Line towards the transceiver IC. Because the desired coupling level is only $25 \mathrm{~dB}$, the coupler is actually designed for much higher frequency to minimize the size of the coupler. Thus, the desired weak coupling is achieved. Other end of the calibration line is terminated to a $100 \Omega$ resistor. The directional coupler also introduces insertion loss for the Main RF Line signal path but due to weak coupling, the effect is negligible.

Fig. $3 b$ shows two of the calibration lines, calibration feeding via, and feeding vias for Main RF Lines. Calibration feeding port location has an offset so that RF-IC can be placed in the middle of the board and IC connections can be as close to the via feeds as possible.

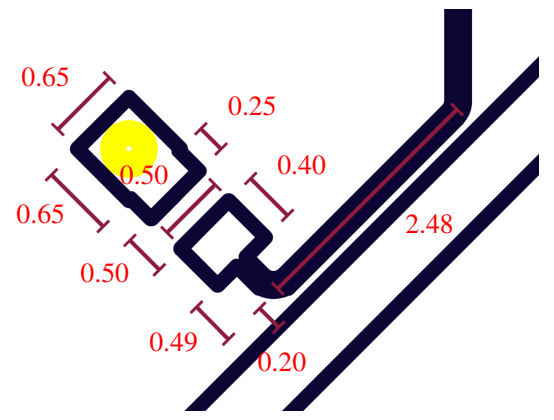

(a)

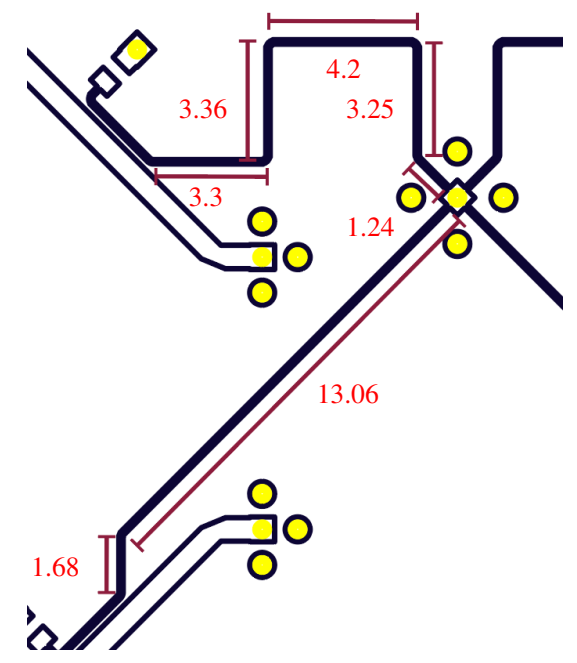

(b)

Figure 3. Calibration network directional coupler shown in (a) and calibration network in (b). All dimensions are in $\mathrm{mm}$.

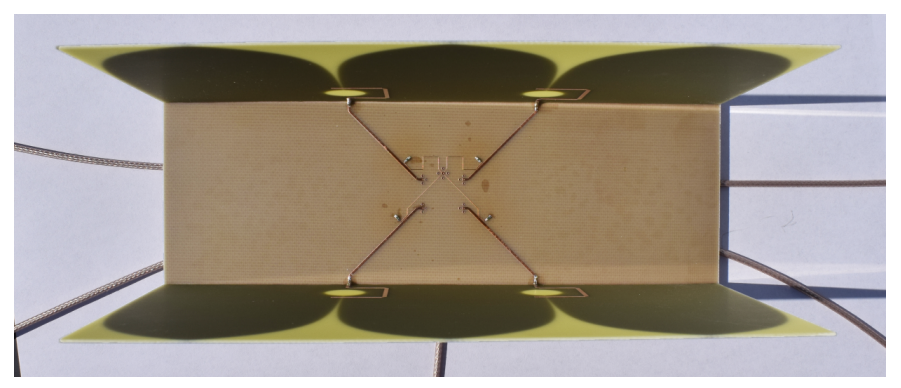

Figure 4. Prototype of the proposed design.

\section{RESULTS}

The combined structure was constructed by soldering two antenna PCBs to the calibration board. Antenna PCBs are mirror images of each other so that the polarity of the antenna remains the same. Fig. 4 shows the prototype of the design. The RF trace side of the calibration board and the balun side of both antenna PCBs are shown in the figure. Five measurement cables are under the structure.

Simulations are done with CST MWS. Measurements are done with two port vector network analyzer (VNA) and by terminating rest of the ports to $50 \Omega$ impedance during the 


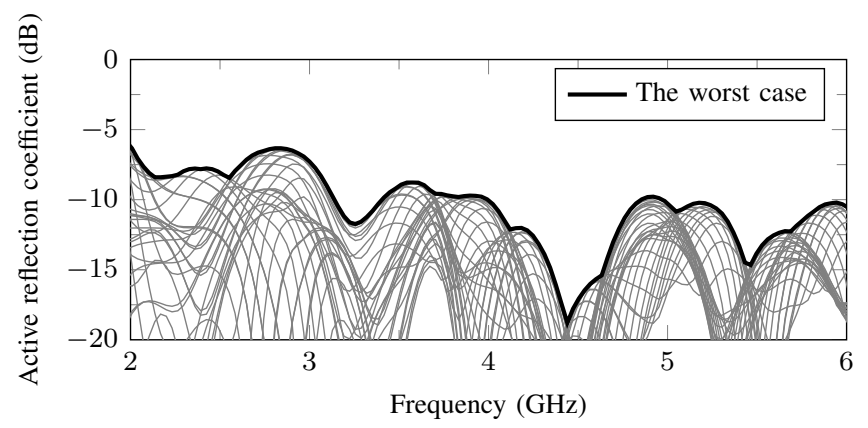

Figure 5. The worst case active reflection coefficient (dB).

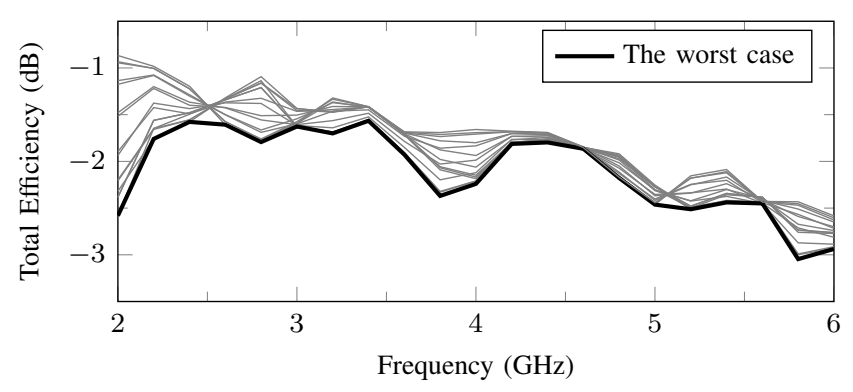

Figure 6. The worst case total efficiency $(\mathrm{dB})$.

measurements. $100 \Omega$ resistors in the design and calibration kit are Vishay FC0402 [10].

\section{A. Active reflection coefficient}

Fig. 5 shows the active reflection coefficient (ARC) in several use cases, when all ports are in the same phase and when the beam is steered. Phase difference of 0 to 210 degrees is studied in 30 degree intervals. The black line shows the envelope curve that represents the worst case ARC over all simulated phase shifts.

The ARC is lower than $-6.3 \mathrm{~dB}$ from 2.0 to $4.0 \mathrm{GHz}$ and below $-9.8 \mathrm{~dB}$ from 4.0 to $6.0 \mathrm{GHz}$. The simulations show that the ARC is heavily dependent on the phase difference between the feed ports and the performance varies when the beam is steered being still at acceptable level.

\section{B. Total efficiency}

Total efficiency is simulated with same setup as the ARC. Fig. 6 shows that the total efficiency of the proposed structure is better than $-3 \mathrm{~dB}$. There is a notable trend of decreasing total efficiency as a function of increasing frequency. This is likely due to FR-4 substrate used in PCB [11]. In addition, the change in the phase of the RF ports affects the total efficiency of the design. Variation between the worst and the best case is the largest at the lowest design frequency.

\section{Calibration network performance}

The transmission coefficients from the calibration network were simulated and measured from the prototype. The results are shown in Fig. 7. Measured responses correspond well to each other over the whole frequency range. Both the simulated

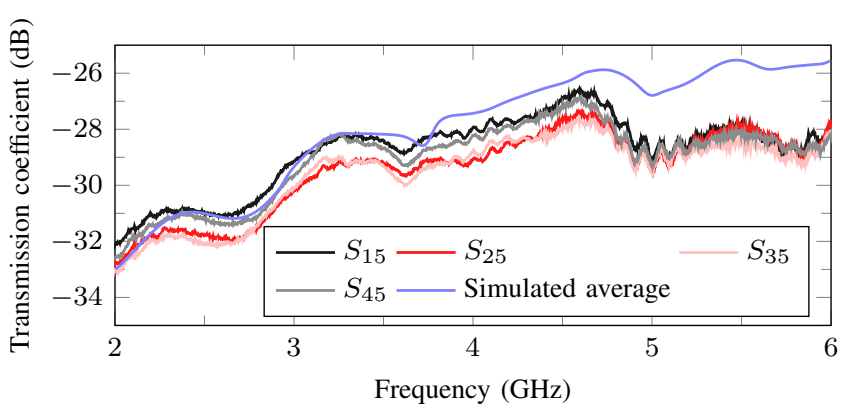

(a)

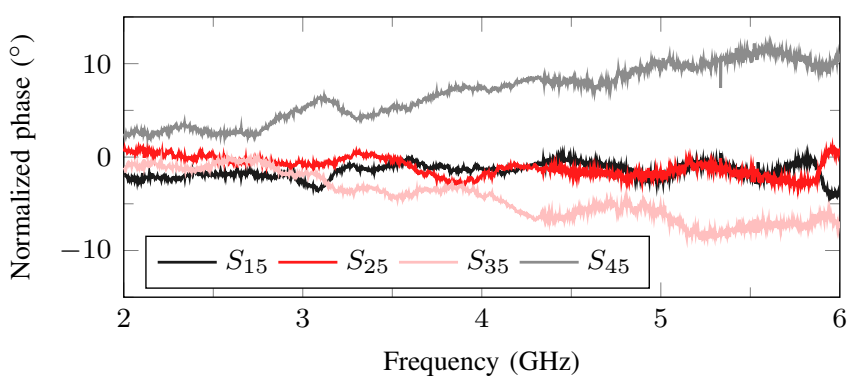

(b)

Figure 7. The measured calibration network performance in transmission coefficients (a) and phase normalized to average phase (b).

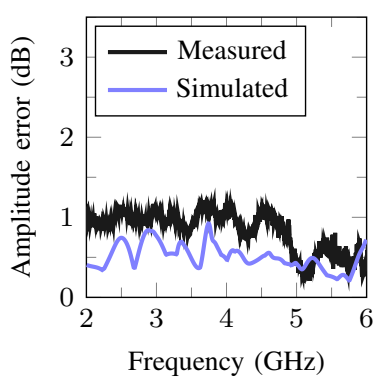

(a)

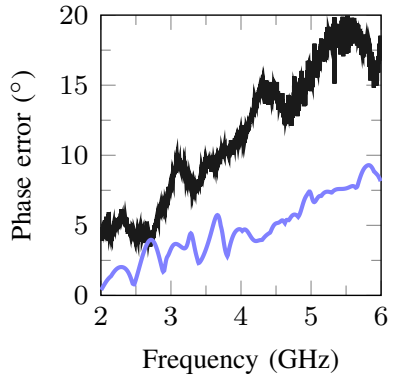

(b)
Figure 8. Differences of the measured transmission coefficients in amplitude (a) and phase (b) response.

and measured amplitude responses in Fig. 7a have a similar trend up to $3.5 \mathrm{GHz}$. At higher frequencies, the difference between simulated and measured results increases. $S_{15}$ and $S_{45}$ are $1 \mathrm{~dB}$ higher than $S_{25}$ and $S_{35}$. This is likely result of three additional $90^{\circ}$ corners in $S_{25}$ and $S_{35}$ path calibration network traces.

The phase of the transmission coefficient is shown in Fig. 7b. These values are normalized to the average phase of all paths. The relative phase response of all paths over the designed frequency range is steady. $S_{15}$ and $S_{25}$ are very similar. However $S_{35}$ and $S_{45}$ have frequency dependent drift. Physically $S_{15}$ and $S_{45}$ are mirror images of each other. This is also the case with $S_{25}$ and $S_{35}$. There is a change in slope between mirrored structures. The differences can be explained with small variations of length in the measurement cables soldered to the PCB. At $6 \mathrm{GHz}$ a small difference 


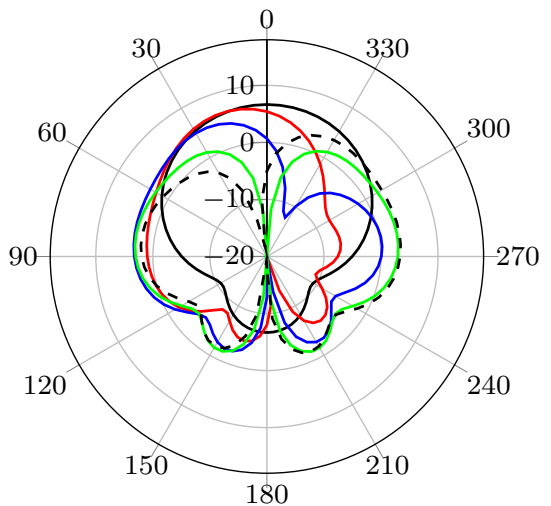

(a) E-plane (Phi=90) at $2 \mathrm{GHz}$.

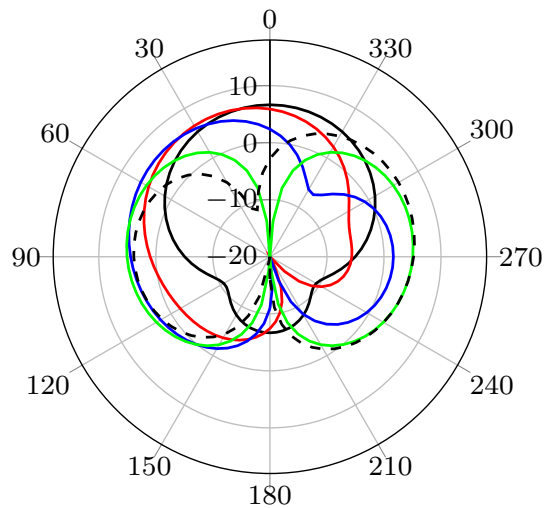

(d) H-plane (Phi=0) at $2 \mathrm{GHz}$.

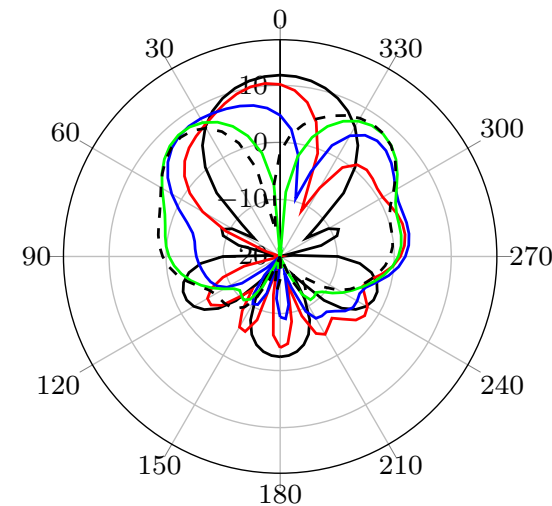

(b) E-plane (Phi=90) at $4 \mathrm{GHz}$.

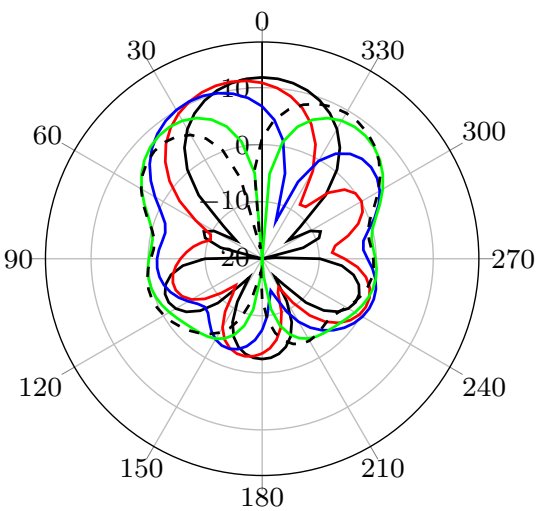

(e) $\mathrm{H}$-plane $(\mathrm{Phi}=0)$ at $4 \mathrm{GHz}$.

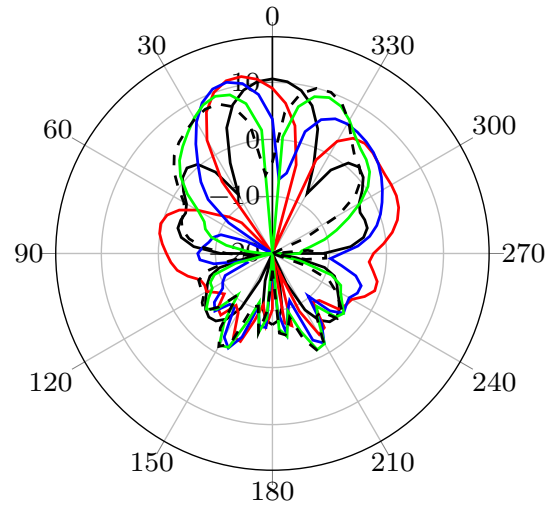

(c) E-plane $(\mathrm{Phi}=90)$ at $6 \mathrm{GHz}$.

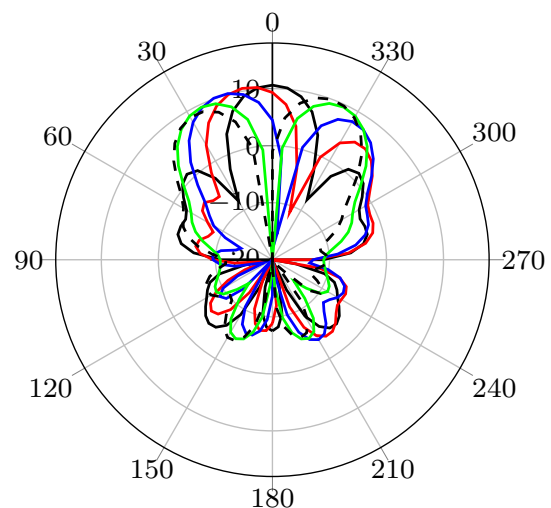

(f) $\mathrm{H}$-plane $(\mathrm{Phi}=0)$ at $6 \mathrm{GHz}$.

$-0^{\circ}-60^{\circ}-120^{\circ}-180^{\circ}---210^{\circ}$

Figure 9. 2D cuts of E-plane and H-plane far fields.

in measurement cable length can lead to noticeable phase difference.

The amplitude and phase errors are calculated from Fig. 7 as a difference between the highest and the lowest responses. Fig. 8 analyzes these amplitude and phase errors between simulated and measured prototypes. The amplitude error in Fig. 8a is $1 \mathrm{~dB}$ over majority of the design frequency range. The phase error of the measured response in Fig. $8 \mathrm{~b}$ is twice the simulated values and ranges from $5^{\circ}$ to $20^{\circ}$.

Based on these results, the design has a working calibration signal distribution network which can be used to calibrate variance between the RF front ends in the transceiver IC. The possible future research on calibration network could include the fine tuning of the calibration network and replacing soldered cables with connectors on PCB to see how high accuracy can be achieved.

\section{Far fields}

The far-field patterns are simulated with CST. The results are represented at three different frequencies to show changes in the far-field with ultra-wide frequency range. The far-fields are shown in E-plane and H-plane cuts in Fig. 9.
Beam steering capability is limited by the emergence of grating lobes caused by $180^{\circ}$ phase shift. Any further beam steering results in a situation where a grating lobe is stronger than the original main lobe. With symmetric far-field, the main lobe is steered $81^{\circ}$ at $2 \mathrm{GHz}$ (Fig. 9a), $38^{\circ}$ at $4 \mathrm{GHz}$ (Fig. 9b), and $21^{\circ}$ at $6 \mathrm{GHz}$ (Fig. 9c) in E-plane. For the $\mathrm{H}$ plane the values are $90^{\circ}$ at $2 \mathrm{GHz}$ (Fig. $9 \mathrm{~d}$ ), $39^{\circ}$ at $4 \mathrm{GHz}$ (Fig. 9e), and $26^{\circ}$ at $6 \mathrm{GHz}$ (Fig. 9f). Therefore the beam steering capability is limited in higher frequencies by the wide relative antenna spacing. We can also see that in H-plane beam direction changes in a linear way as the phase shift is increased. E-plane behavior is not as linear as H-plane. With small phase differences, the beam is steered only a small angle but when the phase shift approaches $150^{\circ}$ the beam steering angle changes rapidly.

Fig. 10 shows the far-field antenna properties as a function of main lobe direction. Fig. 10a shows that the realized gain $\left(G_{r}\right)$ of the array is relatively stable over the whole beam steering range in H-plane. The strongest gain is achieved to broadside and it decreases when the beam is steered. The Eplane response is similar except at $4 \mathrm{GHz}$ where the maximum gain is towards $13^{\circ}$. 


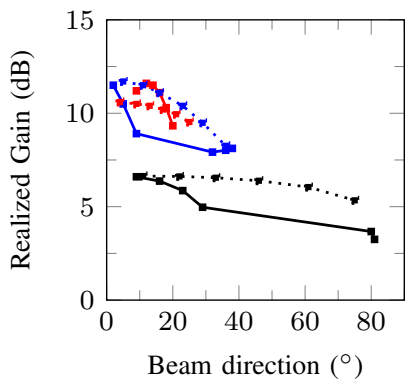

(a)

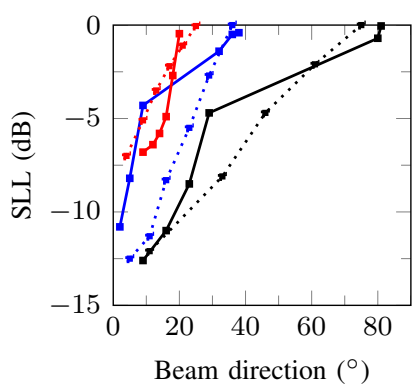

(c)

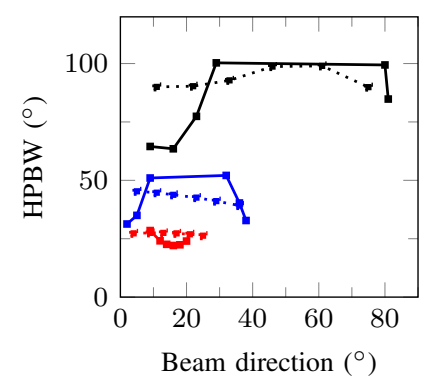

(b)

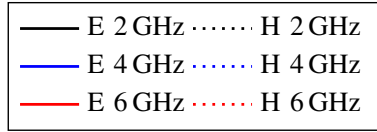

Figure 10. Realized gain (a), half power beam width (b), and sidelobe level (c) are shown as function of peak beam direction.

The obtained half power beam width (HPBW) of the antenna array correlates with theory. HPBW in the lower part of the frequency band is wide $\left(100^{\circ}\right)$ and becomes more narrow as the frequency increases $\left(25^{\circ}\right.$ at $\left.6 \mathrm{GHz}\right)$. There are also differences between E-plane and H-plane HPBW. In H-plane the beam is almost constant when compared to the E-plane. It is obvious that with only two antennas per each steering direction the HPBW remains high in all cases and pencil beam type scanning is not achievable.

The sidelobe level (SLL) of the proposed design is lower than $-10 \mathrm{~dB}$ at $2 \mathrm{GHz}$ and $4 \mathrm{GHz}$ when no or small phase shift is applied. As the beam is steered away from the normal of the array, the SLL in Fig. 10c decreases until the phase shift between feeding ports is $180^{\circ}$. Then the grating lobe equals the main lobe and thus SLL is $0 \mathrm{~dB}$. Similarly at lower frequencies, when beam is steered to side the grating lobes appear and the SLL approaches $0 \mathrm{~dB}$. This behavior is expected due to fixed antenna element distance and large relative difference between the frequency band limits.

\section{CONCLUSION}

A picocell base station antenna array was designed and investigated to harness the potential of the sub- $6 \mathrm{GHz}$ frequency range. A calibration network for IC-transceiver and four element Vivaldi array were designed and successfully combined together. Based on the simulations and measurements, the proposed design provides beam steering capability with ultrawide frequency band. Internal calibration network can be used for transceiver IC calibration.

\section{ACKNOWLEDGMENT}

This paper is part of the 5G TRx project funded by TEKES (The Finnish Funding Agency for Technology and Innovation), Nokia Solutions and Networks, Sasken Finland, Pulse Finland, Huawei Technologies Finland, and RF360.

\section{REFERENCES}

[1] Cisco Systems, Inc., "Cisco visual networking index: Global mobile data traffic forecast update, 20162021 white paper," March 2017.

[2] A. Gupta and R. K. Jha, "A survey of 5G network: Architecture and emerging technologies," IEEE Access, vol. 3, pp. 1206-1232, 2015.

[3] M. M. Kassem and M. K. Marina, "Future wireless spectrum below 6 GHz: A UK perspective," in 2015 IEEE International Symposium on Dynamic Spectrum Access Networks (DySPAN), September 2015, pp. 59-70.

[4] K. Chandra, R. V. Prasad, and I. Niemegeers, "An architectural framework for 5G indoor communications," in 2015 International Wireless Communications and Mobile Computing Conference (IWCMC), August 2015, pp. 1144-1149.

[5] C. R. Anderson and T. S. Rappaport, "In-building wideband partition loss measurements at 2.5 and $60 \mathrm{ghz}$," IEEE Transactions on Wireless Communications, vol. 3, no. 3, pp. 922-928, May 2004.

[6] G. V. Tsoulos and M. A. Beach, "Calibration and linearity issues for an adaptive antenna system," in 1997 IEEE 47th Vehicular Technology Conference. Technology in Motion, vol. 3, May 1997, pp. 1597-1600 vol.3.

[7] P. J. Gibson, "The Vivaldi aerial," in 1979 9th European Microwave Conference, September 1979, pp. 101-105.

[8] M. Barari, A. Tavakoli, and R. Moeini, "Wideband single polarised finite array of Vivaldi antenna," in 2002 9th International Symposium on Antenna Technology and Applied Electromagnetics, July 2002, pp. $1-4$.

[9] B. Shuppert, "Microstrip/slotline transitions: modeling and experimental investigation," IEEE Transactions on Microwave Theory and Techniques, vol. 36, no. 8, pp. 1272-1282, Aug 1988.

[10] Vishay Intertechnology, Inc., High Frequency (up to $40 \mathrm{GHz}$ ) Resistor, Thin Film Surface Mount Chip, April 2017. [Online]. Available: https://www.vishay.com/docs/60093/fcseries.pdf

[11] J. Coonrod, "Understanding when to use FR-4 or high frequency laminates," OnBoard Technology, pp. 26-30, September 2011. 